\title{
Identification of Mutations in the Coding Sequence of the Proto-oncogene c-kit in a Human Mast Cell Leukemia Cell Line Causing Ligand-independent Activation of c-kit Product
}

\author{
Takuma Furitsu, * Tohru Tsujimura, ${ }^{\ddagger}$ Toshiharu Tono, ${ }^{\star}$ Hirokazu Ikeda, * Hitoshi Kitayama, * \\ Uichi Koshimizu," Hiroyuki Sugahara, * Joseph H. Butterfield," Leonie K. Ashman," \\ Yoshio Kanayama, * Yuji Matsuzawa, * Yukihiko Kitamura, ${ }^{*}$ and Yuzuru Kanakura * \\ * Second Department of Internal Medicine and ${ }^{*}$ Department of Pathology, Osaka University Medical School, Suita 565, Japan; \\ ${ }^{\S}$ Research Institute for Microbial Diseases, Osaka University, Suita 565, Japan; "Department of Internal Medicine, Mayo Clinic, \\ Rochester, Minnesota; and 'Leukaemia Research Unit, Hanson Centre for Cancer Research, Adelaide, South Australia
}

\section{Abstract}

The c-kit proto-oncogene encodes a receptor tyrosine kinase. Binding of c-kit ligand, stem cell factor (SCF) to c-kit receptor (c-kitR) is known to activate c-kitR tyrosine kinase, thereby leading to autophosphorylation of c-kitR on tyrosine and to association of $c-k i t R$ with substrates such as phosphatidylinositol 3-kinase (PI3K). In a human mast cell leukemia cell line HMC-1, c-kitR was found to be constitutively phosphorylated on tyrosine, activated, and associated with PI3K without the addition of SCF. The expression of SCF mRNA transcript in HMC-1 cells was not detectable by means of PCR after reverse transcription (RT-PCR) analysis, suggesting that the constitutive activation of $c-k i t R$ was ligand independent. Sequencing of whole coding region of $\mathrm{c}-k i$ it $\mathrm{cDNA}$ revealed that $\mathrm{c}$-kit genes of HMC-1 cells were composed of a normal, wild-type allele and a mutant allele with two point mutations resulting in intracellular amino acid substitutions of Gly-560 for Val and Val-816 for Asp. Amino acid sequences in the regions of the two mutations are completely conserved in all of mouse, rat, and human c-kit. In order to determine the causal role of these mutations in the constitutive activation, murine c-kit mutants encoding Gly-559 and / or Val-814, corresponding to human Gly-560 and / or Val816, were constructed by site-directed mutagenesis and expressed in a human embryonic kidney cell line, 293T cells. In the transfected cells, both c-kitR (Gly-559, Val-814) and ckitR (Val-814) were abundantly phosphorylated on tyrosine and activated in immune complex kinase reaction in the absence of SCF, whereas tyrosine phosphorylation and activation of c-kitR (Gly-559) or wild-type c-kitR was modest or little, respectively. These results suggest that conversion of Asp-816 to $\mathrm{Val}$ in human $\mathrm{c}-k i t \mathrm{R}$ may be an activating mutation and responsible for the constitutive activation of c-kitR in HMC-1 cells. (J. Clin. Invest. 1993.92:1736-1744.) Key words: protooncogene c-kit $\bullet$ point mutation $\cdot$ leukemia $\bullet$ tyrosine kinase • activation

This work was presented in part as a poster at the 34th Annual Meeting of the American Society of Hematology, Anaheim, CA, December 1992, and was published in abstract form (1992. Blood. 80:302a).

Address correspondence to Dr. Yuzuru Kanakura, Second Department of Internal Medicine, Osaka University Medical School, 2-2, Yamada-oka, Suita 565, Japan.

Received for publication 23 March 1993 and in revised form 1 June 1993.

J. Clin. Invest.

(c) The American Society for Clinical Investigation, Inc.

0021-9738/93/10/1736/09 \$2.00

Volume 92, October 1993, 1736-1744

\section{Introduction}

The proto-oncogene c-kit was identified as the cellular homologue of the oncogene v-kit present in the genome of HardyZuckerman 4-feline sarcoma virus that induces multicentric fibrosarcomas in the domestic cat (1). The proto-oncogene c-kit encodes a transmembrane tyrosine kinase receptor that is a member of receptors for PDGF and CSF-1 $(2,3)$. The characteristic features of the receptor tyrosine kinase subfamily are an extracellular domain made up of five immunoglobulin-like repeats, and an intracellular tyrosine kinase, which is split into two domains by a hydrophilic insert sequence (1-4). The ligand for c-kit product has recently been cloned and variously designated as stem cell factor (SCF), ${ }^{1}$ mast cell growth factor, kit ligand, or steel factor (5-12).

The c-kit gene is allelic with the dominant white spotting $(W)$ locus on mouse chromosome $5(13,14)$, whereas the gene encoding SCF resides at steel $(S 1)$ locus on mouse chromosome $10(7,9,12)$. Mutations at either the $W / c-k i t$ locus or the $S 1 /$ SCF locus affect various aspects of hematopoiesis and the proliferation and/or migration of primordial germ cells and melanoblasts during embryogenesis (4, 15-17). In hematopoiesis, both $W / \mathrm{c}-k i t$ and S1/SCF mutations affect the hematopoietic stem cell compartment and give rise to hypoplastic anemia and a deletion of mast cells (4, 15-17). Recent experiments have shown that c-kit product is expressed on mast cells $(4,16,18)$ and on a small, progenitor-cell fraction ( $\sim 4 \%$ ) of adult bone marrow cells (19-21), and that SCF acts synergistically with other hematopoietic growth factors to stimulate the growth of a variety of progenitor cells (5, 6, 22-24).

In addition to normal hematopoietic cells, we and others have investigated the expression and function of c-kit product in human leukemia cells (25-32). In human leukemia cell lines, the expression of c-kit product was detected in erythroleukemia cell lines (HEL, LAMA, and TF-1), megakaryocytic leukemia cell lines (M07E and MEG-01), and mast-cell leukemia cell line (HMC-1 ) $(25,27,31,32)$. Also, c-kit product was found to be expressed in most cases of human acute myeloblastic leukemia (AML) and in some cases of chronic myelogenous leukemia (CML) in blastic crisis $(26,28-31)$. Further, SCF induced rapid tyrosine phosphorylation and activation of c-kit product, leading to proliferation of M07E cells and blast cells in a substantial fraction of AML cases $(27,28)$. These results

1. Abbreviations used in this paper: AML, acute myeloblastic leukemia; CML, chronic myelogenous leukemia; HMC-1, human mast-cell leukemia cell line-1; PI3K, phosphatidylinositol 3-kinase; rh, recombinant human; RTK, receptor tyrosine kinase; S1, steel locus; SCF, stem cell factor; $W$, white spotting locus. 
suggest that c-kit activation may be involved in some aspect of excessive proliferation and aberrant differentiation of human leukemia cells. However, structural abnormalities of c-kit gene contributing to constitutive, ligand-independent activation of the c-kit R kinase have not been reported.

In this study, we have investigated the state of tyrosine phosphorylation and activation of c-kit product in human leukemia cells, and found that, regardless of stimulation with SCF, c-kit product is constitutively phosphorylated on tyrosine residues in HMC-1. Neither SCF mRNA transcript nor biological activity of SCF were detectable from HMC-1 cells, suggesting that the activation of c-kit was not due to autocrine mechanism. The sequence of the c-kit gene revealed unique, as-yetunidentified, two-point mutations in the cytoplasmic domain, resulting in amino acid substitutions of Gly for Val in codon 560 and Val for Asp in codon 816. Further, introduction of the mutated c-kit genes into cells of a human embryonic kidney cell line, 293T, resulted in the generation of the c-kit-encoded protein that was constitutively phosphorylated on tyrosine and activated without the addition of SCF. Thus, our data provide the first evidence that point mutation(s) in c-kit gene is capable of inducing constitutive activation of c-kit product, and raise the possibility that the activating mutation(s) described here may be involved in oncogenesis of some cell types, including mast cells and hematopoietic stem cells.

\section{Methods}

Reagents. Highly purified recombinant human (rh) GM-CSF (rhGMCSF) was a gift from Drs. S. Clark and G. Wong (Genetic Institute, Cambridge, MA). rhSCF was generously provided by Dr. K. M. Zsebo (Amgen Inc., Thousand Oaks, CA). The anti-phosphotyrosine antibody, a murine mAb generated against phosphotyramine, was generously supplied by Dr. B. Druker (Dana-Farber Cancer Institute, Boston, MA). The specificity of the anti-phosphotyrosine mAb was described previously $(33,34)$. Rabbit anti-human c-kit serum was kindly provided by Dr. A. Ullrich (Max-Planck Institute für Biochemie, Martinsried, Germany). This antiserum was generated against a synthetic peptide corresponding to $\mathrm{COOH}$-terminal 16 amino acid residues of c-kitR (2). A murine mAb (YB5.B8) that reacts with the extracellular domain of human c-kit protein was originally raised against human AML blast cells $(21,35)$. The rabbit antiserum against the whole murine c-kit protein (36) was generously donated by Dr. P. Besmer (Cornell University Graduate School of Medical Science, New York ). A rat $\mathrm{mAb}$ (ACK-2) against the extracellular domain of murine c-kit product (19) and the full length of murine c-kit cDNA clone were generously donated by Dr. S-I. Nishikawa (Kumamoto University, Kumamoto, Japan). Rabbit anti-phosphatidylinositol 3-kinase (PI3K) serum was purchased from Upstate Biotechnology, Inc. (Lake Placid, NY); the anti-PI3K serum recognizes an $85-\mathrm{kD}$ subunit of PI3K. Chemically defined serum-free medium (ASF-102) was purchased from Ajinomoto ( Tokyo, Japan ); it contains human transferrin, insulin, and BSA.

Cell lines. HMC-1, a human mast cell leukemia cell line, established from the peripheral blood from a patient with mast cell leukemia, was grown in Iscove's modified Dulbecco's medium supplemented with $10 \%$ horse serum (Flow Lab, North Ryde, Australia) as described previously (37). M07E, a human GM-CSF and IL-3 dependent cell line, was obtained from Dr. Steve Clark, Genetics Institute, and was originally established by Avanzi et al. from the peripheral blood from an infant with acute megakaryocytic leukemia (38). M07E cells were cultured in RPMI-1640 medium (Nacalai Tesque, Kyoto, Japan) supplemented with $10 \%$ FCS (Flow Lab) and $10 \mathrm{ng} / \mathrm{ml}$ rhGMCSF. HEL, a human erythroleukemia cell line, and NTI-4, human fetus-derived fibroblast cell line, were obtained from the Japanese
Cancer Research Resources Bank, and this cell line were adapted to grow and maintained in RPMI 1640 supplemented with $10 \%$ FCS. A human embryonic kidney cell line, 293T was kindly provided by Dr. D. Baltimore (The Rockefeller University, New York), and was derived from human embryonic kidney cells transformed by DNA from human adenovirus type 5 (39). 293T cells were maintained in DMEM (Nacalai Tesque) supplemented with $10 \%$ FCS. In a preliminary study, 293T cells were found to be capable of expressing a greater amount of c-kit product than COS cells after transfection of c-kit gene.

Flow cytometry. Cells $\left(5 \times 10^{5}\right)$ were washed with PBS and resuspended in PBS containing 0.5\% BSA (Sigma Chemical, St. Louis, $\mathrm{MO}), 0.1 \% \mathrm{NaN}_{3}$, and human $\mathrm{Ig}(1 \mathrm{mg} / \mathrm{ml})$ to block binding of antibodies to Fc receptors on the cells. Cells were incubated with YB5.B8 or mouse monoclonal $\mathrm{IgG}_{1}$ control (Becton Dickinson and Co., Mountain View, CA) at $4^{\circ} \mathrm{C}$ for $30 \mathrm{~min}$, then washed three times with the buffer. Cells were subsequently incubated with fluorescein-conjugated goat $\left(\mathrm{Fab}^{\prime}\right)_{2}$ anti-mouse IgG antibody ( Tago, Inc., Burlingame, CA ) at $4^{\circ} \mathrm{C}$ for $30 \mathrm{~min}$, and washed twice before analysis using a FACScan ${ }^{\oplus}$ flow cytometer (Becton Dickinson and Co.).

Immunoblotting. The procedures of cell lysis, gel electrophoresis, and immunoblotting were performed according to the methods described previously $(27,28,33,34)$. Briefly, exponentially growing cells were washed free of serum and growth factors and incubated in serumfree ASF-102 medium for $16 \mathrm{~h}$ at $37^{\circ} \mathrm{C}$ to factor-deprive the cells. The cells ( $10^{7}$ cells suspended in $1 \mathrm{ml}$ of ASF-102 medium) were then exposed to $\mathrm{rhSCF}(100 \mathrm{ng} / \mathrm{ml})$ for $15 \mathrm{~min}$ at $37^{\circ} \mathrm{C}$. After stimulation, cells were washed with cold PBS and lysed in NP-40 buffer $(20 \mathrm{mM}$ Tris- $\mathrm{HCl} \mathrm{pH} \mathrm{8.0,137} \mathrm{mM} \mathrm{NaCl,} 10 \%$ glycerol, $1 \%$ NP-40) containing protease and phosphatase inhibitors at $4^{\circ} \mathrm{C}$ for $20 \mathrm{~min}$. In some experiments, lysis buffer containing $1 \%$ digitonin (Wako Pure Chemical Industries, Ltd., Osaka, Japan ) instead of $1 \%$ NP-40 was used to examine the association of c-kit product with PI3K. Insoluble material was removed by centrifugation at $10,000 \mathrm{~g}$ for $15 \mathrm{~min}$ at $4^{\circ} \mathrm{C}$, and cell lysates were subjected to SDS-PAGE. Proteins were electrophoretically transferred from the gel onto a polyvinylidene difluoride membrane (Immobilon; Millipore Corp., Bedford, MA), and immunoblotting was performed with either anti-phosphotyrosine $\mathrm{mAb}$ or anti-c-kit serum.

For immunoprecipitation, the lysates from rhSCF-stimulated or unstimulated cells were precleared with protein A-Sepharose beads (Pharmacia AB, Uppsala, Sweden) for $2 \mathrm{~h}$ at $4^{\circ} \mathrm{C}$. The precleared lysates were then incubated with a YB5.B8 $\mathrm{mAb}$ and protein A-Sepharose beads to collect the antigen-antibody complexes. The immunoprecipitates were washed five times with lysis buffer containing protease and phosphatase inhibitors, and subjected to SDS-PAGE and immunoblotting with either anti-phosphotyrosine $\mathrm{mAb}$, anti-c-kit serum, or anti-PI3K serum.

Immune complex kinase assay. The cell lysates were prepared from rhSCF-unstimulated cells using NP-40 lysis buffer. The product of c-kit was immunoprecipitated with the YB5.B8 $\mathrm{mAb}$ and protein A-Sepharose beads, and the immune complexes were washed once with PBS,

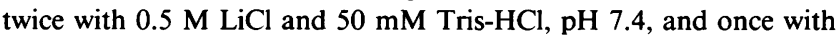
kinase buffer ( $10 \mathrm{mM} \mathrm{MnCl}_{2}, 20 \mathrm{mM}$ Tris- $\mathrm{HCl}, \mathrm{pH} \mathrm{7.4)}$ at $4^{\circ} \mathrm{C}$. Then, the immune complexes were incubated in kinase buffer containing 10 $\mu \mathrm{Ci} / \mathrm{ml}\left[\gamma^{-32} \mathrm{P}\right] \mathrm{ATP}$ (DuPont/NEN Research Products, Boston, MA) for $20 \mathrm{~min}$ at $25^{\circ} \mathrm{C}$, washed, and separated by SDS-PAGE. The gel was dried, and radioactive proteins were detected by autoradiography.

Oligonucleotide primers. PCR was performed by using two complementary oligonucleotide primers for amplifying human SCF cDNA: 5'-ATGAAGAAGACACAACTTG-3' ( 184 to 203 , sense) and 5'-AAGGCATCAATGGATCTATT-3' (615 to 634, antisense). For the amplification of human c-kit cDNA, the following seven oligonucleotide primers were used: primer 1, 5'-GATCCCATCGCAGCTACCGCG-3' ( 1 to 21 , sense); primer 2, 5'-ATCTTCCCCATGATAAACAC-3' (964 to 983 , sense); primer 3 , 5'-AGGAGATAAATGGAAACAATTATGT-3' (1703 to 1727 , sense); primer 4, 5'-TTGAGCATCTTTACAGCGACAGTCA-3' ( 1874 to 1898, antisense); primer 5, 5'-GCCCACCCTGGTCATTACAGAAT-3' (2013 to 2035, sense); primer 6, 5'AAAATCCCATAGGACCAG-3' (2577 to 2594 , antisense ); primer 7 , 
5'-AGTGTGCTCAGAAAGACAGGATTGC-3' (3068 to 3092, antisense). The numbers in parentheses represent the nucleotide numbers on the complementary strands of each cDNA sequence.

cDNA synthesis and PCR amplification. Total cellular RNA was isolated with a guanidine isocyanate method in combination with a cesium chloride modification $(40,41)$. Genomic DNA was prepared from HMC-1 cells by the standard method. Total RNA was converted into cDNA using the primer specific for the subsequent PCR amplification. cDNA synthesized from $4 \mu \mathrm{g}$ of total RNA was amplified in a 100 $\mu l$ reaction mixture by a DNA thermal cycler (Perkin-Elmer Cetus Corp., Norwalk, CT) using Taq DNA polymerase in 25 cycles of 1-min denaturation at $94^{\circ} \mathrm{C}, 2$-min annealing at $55^{\circ} \mathrm{C}$, and 3-min synthesis at $72^{\circ} \mathrm{C}$. To minimize possible artifacts that could be caused by priming with PCR, the number of PCR cycles was kept to a minimum.

Detection of human SCF mRNA transcript by means of PCR after reverse transcription (RT-PCR) analysis. The single-strand cDNA was synthesized from total mRNA of HMC-1 and NTI- 4 cells with both the specific and random primers, and subjected to PCR to amplify the 451-bp DNA fragment between nucleotide 184 and 634 of human SCF cDNA. PCR products were examined on $1 \%$ agarose gel, transferred to nitrocellulose filter (Schleicher \& Schuell, Dassel, Germany), and then hybridized with random ${ }^{32} \mathrm{P}$-labeled probe.

Sequencing of $c$-kit $c D N A$. Sequencing of the whole coding region of c-kit cDNA was performed in HMC-1 cells by using four sets of primers: primers $1-4,2-6,3-6$, and 5-7 as described above. The PCR products amplified by these primers were gel purified, sequentially treated with T4 polynucleotide kinase and the Klenow fragment of DNA polymerase I, and subcloned into the EcoRV site of Bluescript KS(-) (Stratagene Corp., La Jolla, CA). DNA sequences of four subclones obtained from each PCR product were determined and compared with normal sequence of human c-kit cDNA (2). The DNA sequencing reaction was performed by the method of Sanger et al. (42) using $\left[{ }^{32} \mathrm{P}\right] \alpha$-deoxycytidine triphosphate (DuPont/NEN Research Products). In order to further confirm the mutations of c-kit mRNA, the sequencing in the regions of $\mathrm{c}-k i t$ mutations was performed directly using DNA fragments amplified by independent RT-PCR.

Site-directed mutagenesis and transfection. To directly examine the causal role of the c-kit mutations in activation of c-kit tyrosine kinase activity, site-directed mutagenesis was performed using murine c-kit cDNA because of the following reasons. First, amino acid sequences in the regions of the two c-kit mutations are completely conserved between mouse and human. Second, human SCF is known to have little effect on cells expressing murine c-kit receptor (c-kitR) $(6,7)$, whereas murine SCF can exert its actions through interaction with both murine and human c-kitRs $(6,7,27,43)$. We therefore used a combination of murine c-kit cDNA and a human 293T cell line as a transfectant.

The gene encoding murine wild-type c-kit $\mathrm{R}$ was cloned into Bluescript I KS(-) and the orientation of the insert was determined by sequencing. Single-stranded uracil-containing DNA was prepared from Escherichia coli strain CJ236 ( dut $^{-}$ung $^{-}$) as described by Kunkel (44). To generate genes containing G-559 and V-814 mutations, two oligonucleotides of 5'-CAATGGAAGGTTGGCGAGGAGATAAAT$3^{\prime}$ and 5'-GGGCTAGCCAGAGTCATCAGGAATGAT-3' were synthesized, annealed to the template and extended. The double-stranded mismatch containing mutant plasmids were transformed into $\mathrm{ung}^{+} E$. coli strain, and the clones containing each mutation were then screened by sequencing. The DraI-BssHII fragment with G-599 mutation and the NheI-MroI fragment with V-814 mutation were isolated and inserted into the wild type c-kit clone to construct mutants encoding G-559, V-814, or both of them. The full coding sequences of the wildtype and mutated c-kit cDNA were then released by SmaI and HindIII restriction digestion, isolated, and inserted into the expression vector pSV2. The inserts were resequenced to confirm the orientation and mutations within the expression vectors.

The expression vectors containing wild-type and mutated c-kit cDNA were transfected into $293 \mathrm{~T}$ cells by the calcium phosphate method as described previously (45). The cells were cultured in DMEM containing $10 \%$ FCS and fed every $3 \mathrm{~d}$ with fresh medium. To determine the expression of c-kit product in the transfected 293T cells, the transfectants were incubated with $100 \mu \mathrm{Ci} / \mathrm{ml}\left[{ }^{35} \mathrm{~S}\right]$ methionine (DuPont/NEN Research Products) for $6 \mathrm{~h}$, and lysed with NP-40 lysis buffer. The labeled c-kit product was then immunoprecipitated with a rat anti-mouse c-kit mAb (ACK-2) and analyzed by SDS-PAGE and autoradiography. For the analysis of protein tyrosine phosphorylation in the transfectants, cell lysates were subjected to SDS-PAGE and immunoblotting with an anti-phosphotyrosine $\mathrm{mAb}$. To examine the activation and tyrosine phosphorylation of c-kit product, c-kit product of each transfectant was immunoprecipitated with rabbit antiserum against murine c-kit protein from the lysates, and then subjected to in vitro immune complex kinase assay and immunoblotting with an antiphosphotyrosine mAb as described above.

\section{Results}

Constitutive tyrosine phosphorylation of c-kit product in HMC1 cells. The expression of c-kit product was first examined with an anti-c-kit mAb, YB5.B8, which recognizes the extracellular domain of human c-kit product (35). Flow cytometry analysis showed that c-kit product was expressed on HMC-1, M07E, and HEL cells at an almost equal level (Fig. $1 A$ ). Despite the high level of c-kit expression on HMC-1 cells, proliferation of HMC-1 cells was not augmented by the treatment with rhSCF (data not shown), whereas rhSCF was reported to induce proliferation of M07E and HEL cells $(27,31)$.

To examine the impaired effect of rhSCF on proliferation of HMC-1 cells, we investigated the effect of rhSCF on tyrosine kinase activity in HMC-1, M07E, and HEL cells. The cells were removed from growth factors for $16 \mathrm{~h}$ and then stimulated with $100 \mathrm{ng} / \mathrm{ml}$ of rhSCF for $15 \mathrm{~min}$ at $37^{\circ} \mathrm{C}$. Changes in tyrosine phosphorylation were detected by immunoblotting with a $\mathrm{mAb}$ specific for phosphotyrosine. After stimulation of M07E and HEL cells with rhSCF, increased phosphotyrosine was observed in proteins particularly at molecular mass of 145 $\mathrm{kD}$ (Fig. $1 B$ ). In HMC-1 cells, by contrast, tyrosine phosphorylation of $145-\mathrm{kD}$ protein was observed even in the absence of exogenous rhSCF, and rhSCF treatment resulted in only a modest increase in tyrosine phosphorylation of the protein (Fig. $1 B$ ).

In order to determine if the 145 protein was c-kit product, c-kit product was immunoprecipitated and analyzed by immunoblotting with either anti-phosphotyrosine $\mathrm{mAb}$ or anti-c-kit serum. As shown in Fig. $1 C$, tyrosine phosphorylation of c-kit product was detected in HMC-1 cells regardless of rhSCF stimulation, while it was considerably increased by stimulation with rhSCF in M07E and HEL cells.

Activation of c-kit product in HMC-1 cells. Ligand binding to c-kit product is known to activate the c-kit receptor tyrosine kinase, thereby leading to c-kit-tyrosyl autophosphorylation and the association of c-kit with its substrates such as phosphatidylinositol 3-kinase (PI3-kinase) $(4,46-48)$. To determine whether or not the constitutive tyrosine phosphorylation of c-kit product in HMC-1 cells was accompanied by activation of c-kit receptor tyrosine kinase, we examined the association of c-kit product with PI3K and the autokinase activity of c-kit products in the cells.

HMC-1, M07E, and HEL cells were factor starved and exposed to medium alone or rhSCF $(100 \mathrm{ng} / \mathrm{ml})$ for $15 \mathrm{~min}$ at $37^{\circ} \mathrm{C}$, and then lysed with lysis buffer containing $1 \%$ digitonin. Before and after stimulation with rhSCF, c-kit products were immunoprecipitated with a YB5.B8 mAb from the cell lysates and divided into two aliquots, one of which was subjected to 
A

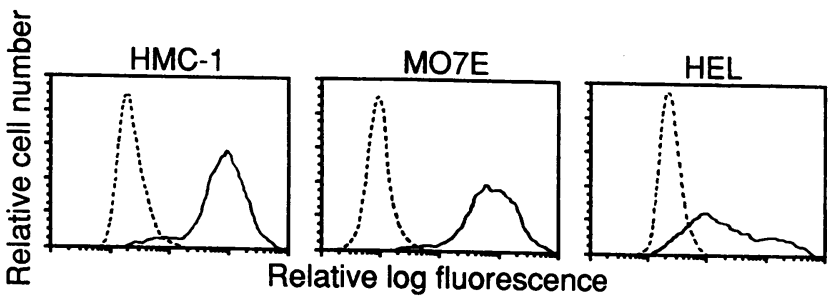

B

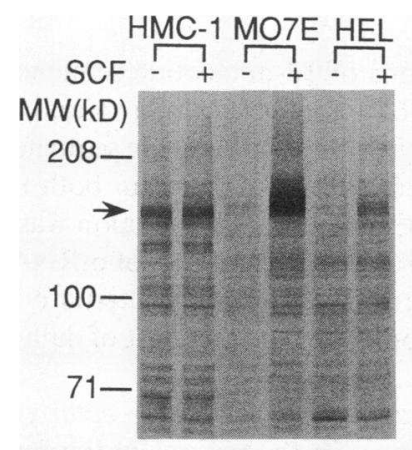

C

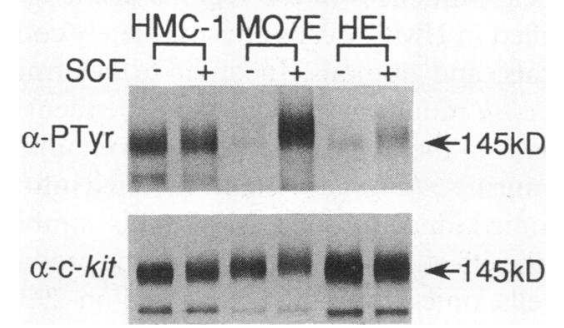

Figure 1. Expression of c-kit product on HMC-1, M07E, and HEL cells $(A)$ and effects of rhSCF on tyrosine phosphorylation of cellular proteins $(B)$ and c-kit product $(C)$ in the cell lines. Flow cytometric analysis of the surface binding of a monoclonal anti-c-kit antibody (YB5.B8) to HMC-1, M07E, and HEL cells $(A)$. Cells were incubated in either negative control antibody (---) or YB5.B8 (-), washed, incubated with fluorescein-conjugated goat $\left(\mathrm{Fab}^{\prime}\right)_{2}$ anti-mouse IgG antibody, and analyzed on a FACScan ${ }^{\oplus}$. For identification of phosphotyrosine-containing proteins, cell lysates were obtained before and after treatment with $\mathrm{rSCCF}(100 \mathrm{ng} / \mathrm{ml})$ for $15 \mathrm{~min}$ at $37^{\circ} \mathrm{C}$ and subjected to immunoblot with anti-phosphotyrosine mAb $(B)$. To examine changes in tyrosine phosphorylation of c-kit product, c-kit product was immunoprecipitated with YB5.B8 from cell lysates before and 15 min after rhSCF stimulation, and immunoblot was performed with either anti-phosphotyrosine mAb or anti-c-kit serum $(C)$.

immunoblotting with anti-PI3K serum and another with antic-kit serum. Based on $\alpha$-c-kit immunoblot data as shown in the lower portion of Fig. 2, approximately equivalent amounts of c-kit products were immunoprecipitated before and after stimulation with rhSCF in each cell line. By contrast, $\alpha$-PI3K immunoblot as shown in the upper portion of Fig. 2 revealed that the $85-\mathrm{kD}$ subunit of PI3K was co-immunoprecipitated with antic-kit antibody only after stimulation with rhSCF in two cell lines, M07E and HEL, whereas it was co-immunoprecipitated in HMC-1 cells even before stimulation with rhSCF.

We next examined the autokinase activity of c-kit products in immune complex kinase assay. Products of c-kit were immunoprecipitated from NP-40 lysates of HMC-1, M07E, and HEL cells before treatment with rhSCF, and assayed for autokinase activity. Tyrosine kinase activity of c-kit products was

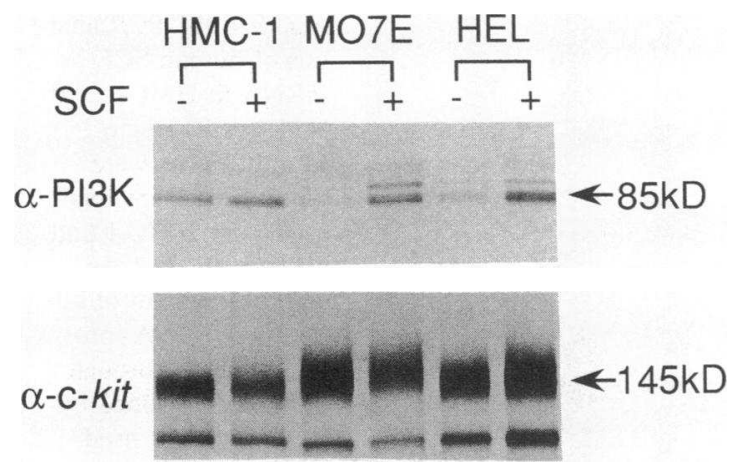

Figure 2. Association of c-kit product with PI3K in HMC-1, M07E, and HEL cells. Cells were treated with $\operatorname{rhSCF}(100 \mathrm{ng} / \mathrm{ml})$ for 15 min at $37^{\circ} \mathrm{C}$ and lysed with $1 \%$ digitonin. c-kit product and c-kit-associated molecules were immunoprecipitated with YB5.B8 and protein A-Sepharose beads from the cell lysates before and after stimulation with rhSCF. The immunoprecipitates were divided into two aliquotes, separated by SDS-PAGE, and subjected to immunoblot analyses of PI3K (upper panel) and c-kit product (lower panel).

readily detectable in HMC-1 cells in the absence of SCF stimulation, whereas it was only faint in M07E or HEL cells (Fig. 3). In this experiment, almost equal amounts of c-kit products were immunoprecipitated in these samples (data not shown).

Absence of SCF MRNA transcripts in HMC-1 cells. One of the possible explanations for the results shown in Figs. 1-3 was that HMC-1 cells might produce SCF. The expression of SCF mRNA transcripts was examined by RT-PCR Southern blot analysis in HMC-1 cells and also in NTI-4 cells that were used as a positive control. As shown in Fig. 4, SCF mRNA transcript was not observed in HMC-1 cells, while a 451-bp fragment of SCF mRNA was easily detected in NTI-4 cells. Further, the culture supernatant of HMC-1 did not have any activities in stimulating proliferation or inducing tyrosine phosphorylation of c-kit product in M07E cells (data not shown). Thus, the constitutive tyrosine phosphorylation and activation of c-kit product in HMC-1 cells were not attributed to autocrine mechanism.

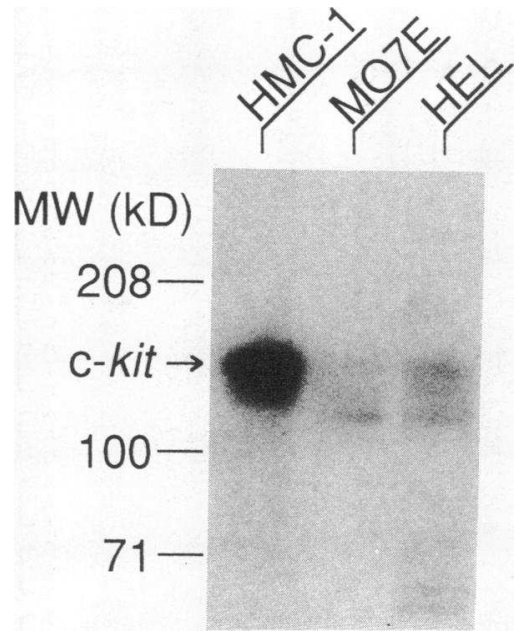

Figure 3. Immune complex kinase assay for ckit tyrosine kinase activity in HMC-1, M07E, and HEL cells. c-kit product was immunoprecipitated with YB5.B8 from NP-40 lysates before stimulation with rhSCF, and the immunoprecipitates were incubated in kinase buffer containing $10 \mu \mathrm{Ci} / \mathrm{ml}\left[\gamma^{-32} \mathrm{P}\right] \mathrm{ATP}$ for $20 \mathrm{~min}$ at $25^{\circ} \mathrm{C}$, washed, and separated by SDS-PAGE. Incorporation of ${ }^{32} \mathrm{P}$ into c-kit product was visualized by autoradiography. In this study, the immunoprecipitates from HMC-1, M07E, and HEL cells contained approximately equivalent levels of c-kit products. 


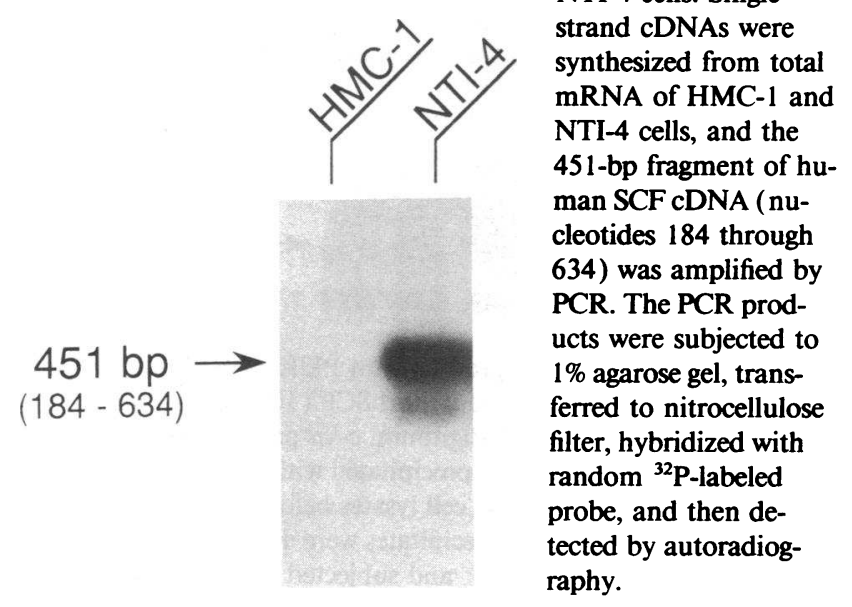

Mutations in c-kit $c D N A$. An alternate mechanism which might explain the constitutive tyrosine phosphorylation and activation of c-kit products in HMC-1 cells would be due to mutations in the c-kit proto-oncogene. Southern blot analyses of genomic DNA from HMC-1 using EcoRI, HindIII, and BamHI showed neither rearrangement nor amplification in ckit gene as compared with normal human placenta (data not shown). However, sequencing of c-kit cDNA demonstrated that c-kit cDNA of HMC-1 cells carries heterozygous transitions in codon 560 (GTT $\rightarrow$ GGT, nucleotides 1699 through 1701 in the region between the membrane and the ATP-binding domains) and codon 816 (GAC $\rightarrow$ GTC, nucleotides 2467 through 2469 in the phosphotransferase domain), which result in the amino acid substitutions of Gly-560 for Val and Val-816 for Asp, respectively. These two point mutations were observed in cDNA clones generated from three independent PCR products, and also confirmed by direct sequencing of PCR products amplified independently (Fig. 5). Further, sequencing of cDNA fragment (nucleotides 964 through 2594) covering the regions of both mutations was performed in four subclones. This analysis showed that two subclones had both mutations, but the remaining two subclones had neither mutation. These results indicate that c-kit genes of HMC-1 cells are composed of a normal wild-type allele and a mutant allele with two point mutations.

In addition, the deletions of 12-nucleotide sequence (nucleotides 1550 through 1561), the extracellular 4-amino acid sequence (Gly-Asn-Asn-Lys), and 3-nucleotide sequence (nucleotides 2163 through 2165 ) were observed in both normal and mutant alleles of HMC-1. The former deletion was previously reported to be due to alternative splicing of mRNA (49); the latter was also considered as the result of alternative splicing of mRNA between exon 14 and 15, in light of data on the human c-kit genome (50).

Mutation(s) leads to activation of $c$-kit receptor tyrosine kinase. Amino acid sequences in the regions of the two c-kit mutations identified in HMC-1 cells are completely conserved in all of mice, rats, and humans. In order to determine the direct role of the c-kit mutations in ligand-independent activation of c-kit products, the expression vectors containing normal or mutated murine c-kit genes were transfected into cells of a human embryonic kidney cell line, 293T. This combination could minimize the effect of SCF, which might be produced by the transfected cells, on c-kit product expressed on 293T cells (see Methods).

We used site-directed mutagenesis to construct mutant murine c-kit $\mathrm{R}$ containing substitutions of Gly for Val at codon 559 and/or Val for Asp at codon 814, which corresponded to Gly-560 and/or Val-816 of mutant human c-kitR, respec-

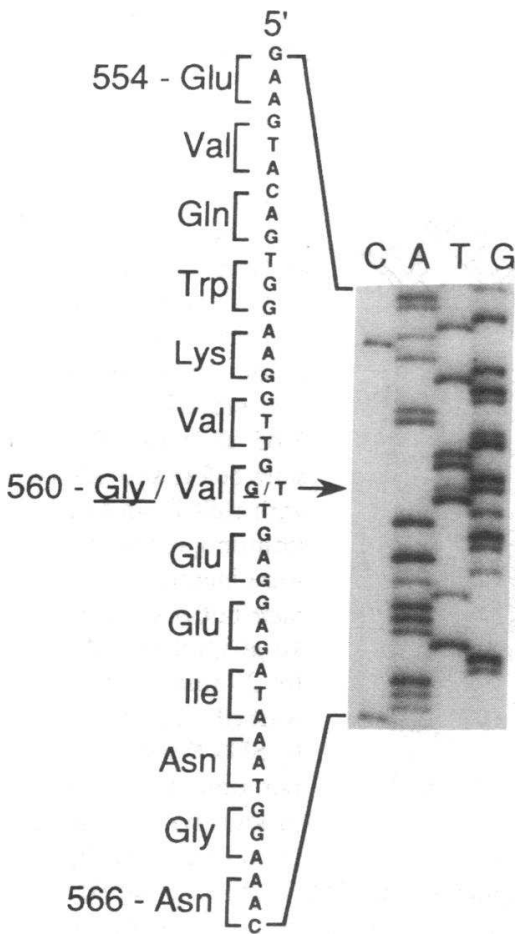

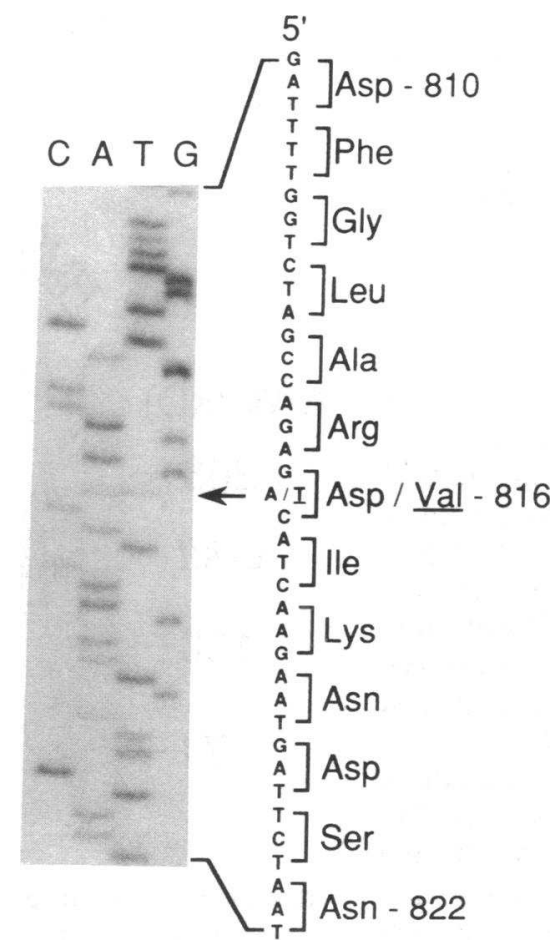

Figure 5. Nucleotide sequence including the mutation sites of c-kit cDNA obtained from HMC-1 cells. Shown are results from direct sequencing of c-kit DNA fragments from amino acid residues 554 to 566 and those 810 to 822 . HMC-1 cells were found to carry heterologous mutations of c$k i t$ gene at codons 560 and 816 . These two mutations were observed in cDNA clones from three independent PCR products, and sequencing of cDNA fragments (nucleotides 964 through 2594) in four clones revealed that the c-kit gene of HMC-1 is composed of a normal and a mutant allele. The arrows indicate the mutation sites, and the altered nucleotides and amino acids are underlined. 

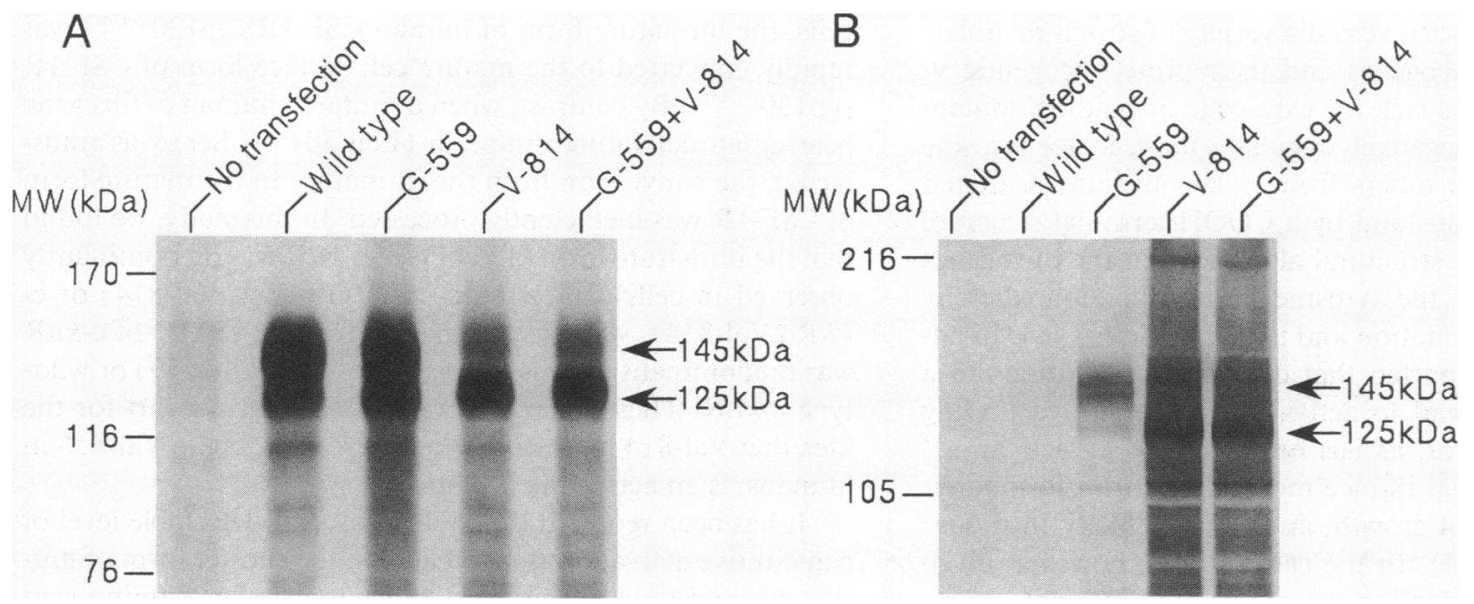

Figure 6. Expression of c-kit product $(A)$ and identification of phosphotyrosine-containing proteins $(B)$ in $293 \mathrm{~T}$ cells transfected with wild-type and mutated murine c-kit genes. The cells were transfected with wild-type or mutated murine c-kit genes encoding wild-type c-kit $\mathrm{R}$ (wild type), c-kitR (Gly-559; G-559), c-kitR (Val-814; V-814), or c-kitR (Gly-559, Val-814; G-559, V814). Also, 293T cells that did not receive transfection were used as a negative control (no transfection). $(A)$ The indicated cells were labeled for $6 \mathrm{~h}$ with $100 \mu \mathrm{Ci} / \mathrm{ml}\left[{ }^{35} \mathrm{~S}\right] \mathrm{methionine}$ and lysed. c-kit product was then immunoprecipitated and analyzed by SDS-PAGE and autoradiography. $(B)$ Lysates of the indicated cells were normalized for protein content, and were subjected to SDS-PAGE and immunoblotting with an anti-phosphotyrosine mAb. The mobilities of the mature $(145-\mathrm{kD})$ and immature $(125-\mathrm{kD})$ forms of $\mathrm{c}-$ kit products are indicated at right.

tively. When wild-type or mutated c-kit genes were transfected in $293 \mathrm{~T}$ cells, the c-kit gene products were synthesized in the cells as 145 - and $125-\mathrm{kD}$ proteins (Fig. $6 \mathrm{~A}$ ), which were the mature and immature forms of c-kit products, respectively (51). Although each transfectant expressed approximately equal amounts of c-kit products, a major c-kit product that incorporated $\left[{ }^{35} \mathrm{~S}\right]$ methionine was the immature form in the cells expressing c-kitR (Gly-559, Val-814) or c-kitR ( Val-814), whereas it was the mature form in the cells expressing c-kit $\mathrm{R}$ (Gly-559) or wild-type c-kitR (Fig. $6 \mathrm{~A}$ ).

Before and after transfection of c-kit genes, changes in tyrosine phosphorylation of proteins were detected by immunoblotting with anti-phosphotyrosine mAb (Fig. 6 B). As compared with nontransfected $293 \mathrm{~T}$ cells, little or no increase in tyrosine phosphorylation was observed in the cells expressing wild-type c-kitR. However, a number of proteins, including 125- and $145-\mathrm{kD}$ proteins, were heavily phosphorylated on tyrosine in the cells expressing c-kitR ( Val-814) or c-kitR (Gly-559, Val814). Also, a modest degree of tyrosine phosphorylation of 125- and $145-\mathrm{kD}$ proteins was noted in the cells expressing c-kitR (Gly-559).
In order to further examine the tyrosine phosphorylation and activation of c-kit products in the transfected cells, c-kit products were immunoprecipitated and assayed by immunoblotting with an anti-phosphotyrosine $\mathrm{mAb}$ and also for autokinase activity. In the absence of SCF, both c-kitR (Val-814) and c-kitR (Gly-559, Val-814) were found to be abundantly phosphorylated on tyrosine, and c-kitR ( Val-814) was also phosphorylated on tyrosine, albeit to a much lesser degree, whereas wild-type c-kitR was not (Fig. $7 \mathrm{~A}$ ). Further, in accord with the findings on the immunoblotting analysis, immune complex kinase assay showed a striking autokinase activity in both c-kitR ( Val-814) and c-kitR (Gly-559, Val-814), a weak activity in c-kitR (Gly-559), and little or no activity in wildtype c-kitR in the absence of SCF (Fig. 7 B).

\section{Discussion}

Many receptor tyrosine kinases were first identified as retroviral oncogenes such as v-erbB, v- $f m s$, and v-kit that encode oncogenic forms of epidermal growth factor R (EGFR), CSF$1 \mathrm{R}$, and $\mathrm{c}-k i t \mathrm{R}$, respectively $(1,46,52)$. Comparative nucleo-

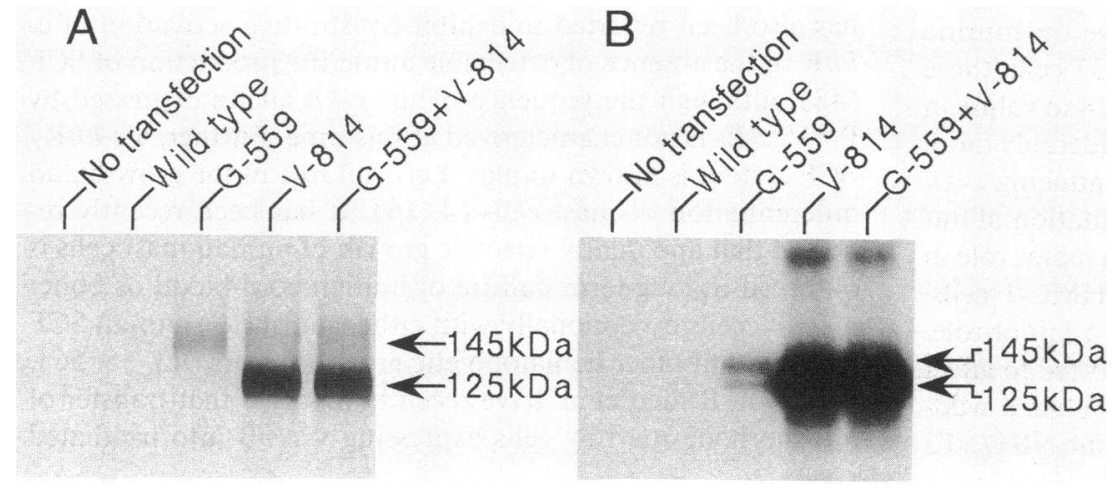

Figure 7. Tyrosine phosphorylation $(A)$ and activation $(B)$ of wild-type and mutated murine c$k i t R$ expressed in 293T cells in the absence of exogenous SCF. $(A)$ c-kit product was immunoprecipitated from the lysates of the indicated cells, and subjected to SDS-PAGE and immunoblotting with an anti-phosphotyrosine $\mathrm{mAb}$. $(B)$ Immune complex kinase assay for c-kit tyrosine kinase activity. c-kit product was immunoprecipitated from lysates from the indicated cells, and the immunoprecipitates were incubated in kinase buffer containing $10 \mu \mathrm{Ci} / \mathrm{ml}\left[\gamma^{32} \mathrm{P}\right] \mathrm{ATP}$ for $20 \mathrm{~min}$ at $25^{\circ} \mathrm{C}$, washed, and separated by SDS-PAGE. Incorporation of ${ }^{32} \mathrm{P}$ into c-kit product was visualized by autoradiography. The mobilities of the mature (145-kD) and immature (125-kD) forms of $\mathrm{c}-k i t$ products are indicated at right. 
tide sequencing analyses revealed a variety of structural differences between the oncogenes and their proto-oncogenes: v$e r b \mathrm{~B}$ and $\mathrm{v}-k i t$ proteins lack the extracellular binding domain of the receptors that negatively regulates the receptor tyrosine kinase activity; v- $f m s$ differs from $\mathrm{c}-\mathrm{fms}$ by nine scattered amino acid substitutions and by a $\mathrm{COOH}$-terminal structure $(3,46,53,54)$. These structural alterations in the oncogenes activate constitutively the tyrosine kinase function, thereby leading to cell transformation and tumorigenicity (46). In addition, it has been reported that even point mutations in a proto-oncogene can lead to activation of the corresponding tyrosine kinase receptor, as has been shown by "activating" mutations of c- $f m s$ that induce morphologic transformation, anchorage-independent growth, and tumorgenicity in mouse NIH 3 T3 cells $(53,54)$. In the case of c-kit, however, all of mutations so far described in mice, rats, and humans are reported to affect either the structure of c-kit or c-kit expression, resulting in a decrease in c-kit tyrosine kinase activity (4).

In this study, we have shown that c-kit product is constitutively phosphorylated on tyrosine and activated in HMC-1 cells even in the absence of SCF-stimulation. Further, c-kit product was found to be associated with PI3K without the exogenous SCF. The ligand-induced association of c-kit product with PI3K is reported to depend on autophosphorylation of c-kit product on tyrosine residues where the association occurs (48). Therefore, tyrosine residues that are constitutively phosphorylated in HMC-1 cells may be indistinguishable from those phosphorylated by ligand stimulation. However, neither mRNA transcript nor biological activity of SCF were detectable from HMC-1 cells. These results suggest that c-kit product in HMC-1 cells is constitutively activated in a ligand-independent manner.

Sequencing analyses have revealed that c-kit genes of HMC-1 cells are composed of a normal, wild-type allele and a mutant allele with two point mutations resulting in amino acid substitutions of Gly-560 for Val and Val-816 for Asp in the intracellular domain. In order to analyze the effect of these previously unidentified substitutions on c-kit kinase function, mutated murine c-kit genes encoding murine c-kitR (Gly-559 and/or Val-814), which were constructed by site-directed mutagenesis to correspond to human c-kitR (Gly-560 and/or Val816), were transfected in a human embryonic kidney cell line, $293 \mathrm{~T}$ cells. In the transfected cells, wild-type c-kitR was neither phosphorylated on tyrosine in vivo nor activated in immune complex kinase reaction in the absence of SCF. By contrast, both c-kitR (Gly-559, Val-814) and c-kitR (Val-814) were strikingly phosphorylated on tyrosine and activated in the absence of SCF. Further, c-kitR (Gly-559) was also phosphorylated on tyrosine and activated, albeit to a much lesser degree. Given the fact that human SCF is hardly active on murine c-kit $\mathrm{R}(6,7)$ even if it might be produced from $293 \mathrm{~T}$ cells, these results indicate that conversion of aspartic acid-814 to valine in murine $\mathrm{c}-k i t \mathrm{R}$ is an activating mutation and considerably more efficient than that of valine-559 to glycine in inducing c-kit kinase activity. It is therefore suggested that a mutation of human c-kit gene at codon 816 (Asp $\rightarrow$ Val ) plays a major role in the constitutive activation of c-kit product in HMC-1 cells, while a mutation at codon 560 ( Val $\rightarrow$ Gly) has a minor role.

The activating mutation of $\mathrm{c}-\mathrm{fms}$ gene is reported to affect intracellular transport of human CSF-1R (54). When a wildtype human c- $f m s$ gene was transfected into murine NIH/3T3 cells, the immature form of human CSF-1R (gp130 c-fms $)$ was rapidly converted to the mature cell surface form of CSF-1R $\left(\mathrm{gp} 150^{\mathrm{c}-f m s}\right)$. By contrast, when a mutated human c- $f m s$ gene bearing an activating mutation (Leu-301 $\rightarrow$ Ser) was transfected, the conversion from the immature to the mature form of CSF-1R was inefficiently processed. In this study, we found that the immature form ( $125 \mathrm{kD}$ ) of c-kit $\mathrm{R}$ was predominantly observed in cells expressing c-kitR (Gly-559, Val-814) or ckitR (Val-814), whereas the mature form (145 kD) of c-kit $\mathrm{R}$ was predominant in cells expressing c-kitR (Gly-559) or wildtype $\mathrm{c}-k i t \mathrm{R}$. These results provide additional support for the idea that Val-814 in murine c-kit, corresponding to Val-816 in humans, is an activating mutation.

It has been reported that only a low but detectable level of constitutive activation is noticeable in the shorter form of murine c-kit product, which lacks the extracellular 4 amino acid sequence (Gly-Asn-Asn-Lys) due to the alternative splicing of mRNA (49). This shorter form of c-kit is found in the half of normal murine or rat c-kit cDNA clones $(40,49)$. Also in HMC-1 cells, we found that the half of c-kit cDNA clones lacks the same four-amino acid sequence in both normal and mutated c-kit genes. In order to compare c-kitR (Gly-559 and/or Val-814) with the shorter form of wild-type c-kitR and to determine the direct role of the c-kit mutations in the constitutive activation, we used the shorter form of wild-type murine c-kit cDNA as a template for the site-directed mutagenesis in this study. In the transfected $293 \mathrm{~T}$ cells, tyrosine phosphorylation and activation were barely detectable in the shorter form of $\mathrm{c}-k i t \mathrm{R}$ which was used as a normal control, whereas they were readily detectable in c-kitR (Gly-559 and/or Val-814). These results indicate that c-kit mutations identified in this study are considerably more active than the shorter form of c-kit, and that the shorter form is unlikely to contribute to the constitutive activation of c-kit product in HMC-1 cells.

Although the mutations described here are found to lead to the constitutive activation of c-kit tyrosine kinase activity, the role of the c-kit activating mutations in leukemogenesis remains to be elucidated. In a previous study, we showed that c-kit product was phosphorylated on tyrosine in freshly prepared leukemic cells of some AML cases even in the absence of SCF stimulation (28). However, occurrences of tyrosine phosphorylation of c-kit product observed in the AML cases were not so striking as that in HMC-1 cells (28). Therefore, an activating mutation at residue 816 (Asp $\rightarrow$ Val) may not occur frequently in de novo AML, while it is still possible that certain mutations of $\mathrm{c}-k i t \mathrm{R}$ other than $\mathrm{c}-k i t \mathrm{R}$ ( Val-816) may contribute to the modest degree of c-kit activation in AML cells. It is interesting to note that HMC-1 is a human mast-cell leukemia cell line (37), and that a mouse mastocytoma cell line, P-815, has also been reported to exhibit constitutive activation of c$k i t \mathrm{R}$ in the absence of detectable autocrine production of SCF (48), although the sequence of the c-kit alleles expressed by P-815 cells is not characterized at this time. Further, a c-kitR/ SCF system is known to play a crucial role in the growth and differentiation of mast cells $(4,16)$. It has been recently reported that apparently selective growth of human mast cells is observed in long-term culture of human cord-blood or bonemarrow cells exceptionally with either murine or human SCF but not with other hematopoietic growth factors $(43,55,56)$. Also, von Ruden et al. have recently reported that transfer of murine bone marrow cells expressing $\mathrm{v}$-erbB into irradiated 
mice results in the development of lethal systemic mast cell leukemia, and suggested that v-erbB may use parts of a signal transduction pathway normally coupled to c-kit $R$ (57). These findings raise the possibility that activating mutations of c-kit described here may be involved in neoplastic transformation of mast cells, although it is as yet unclear whether these mutations by themselves can lead to factor-independent growth and tumorgenicity. It would be of considerable interest to examine whether the transfection of nontumorigenic or factor-dependent cell lines with the mutant forms of c-kit genes results in the production of a tumorigenic or factor-independent phenotype.

In addition to hematological malignancies, expression of c-kit proto-oncogene has been found in various tumors such as lung cancer and germ cell tumors $(58,59)$. Further, the amino acid sequence in a region of the kinase domain containing Asp816 of human c-kit product is widely conserved in many other RTKs such as receptors for PDGF, CSF-1, and insulin (60). Therefore, it is possible that the unique activating mutation in codon 816 of human c-kitR, or a similar mutation in other RTKs, may participate in the development of a variety of malignancies. Further studies on c-kit mutants will be necessary to understand the mechanisms by which growth factor receptors influence oncogenesis and normal growth control.

\section{Acknowledgments}

We would like to thank Dr. K. M. Zsebo for generously providing rhSCF, Dr. A. Ullrich for rabbit anti-human c-kit serum, Dr. P. Besmer for rabbit anti-murine c-kit serum, Dr. S-I. Nishikawa for rat anti-murine c-kit mAb (ACK-2) and full length of murine c-kit cDNA clone, and Dr. D. Baltimore for 293T cell line.

This work was supported in part by grants from the Ministry of Education, Science and Culture of Japan, the Research Foundation for Cancer and Cardiovascular Diseases (Osaka), and the Inamori Foundation.

\section{References}

1. Besmer, P., J. E. Murphy, P. C. George, F. Qui, P. J. Bergold, L. Lederman, H. W. Snyder Jr., D. Brodeur, E. E. Zukerman, and W. D. Hardy. 1986. A new acute transforming feline retrovirus and relationship of its oncogene v-kit with the protein kinase gene family. Nature (Lond.). 320:415-421.

2. Yarden, Y., W. J. Kuang, T. Yang-Feng, L. Coussens, S. Munemitsu, T. J. Dull, E. Chen, J. Schlessinger, U. Francke, and A. Ullrich. 1987. Human protooncogene c-kit: a new cell surface receptor tyrosine kinase for an unidentified ligand. EMBO (Eur. Mol. Biol. Organ.) J. 6:3341-3351.

3. Qiu, F. H., P. Ray, K. Brown, P. E. Barker, S. Jhanwar, F. H. Ruddle, and P. Besmer. 1988. Primary structure of c-kit: relationship with the CSF-1/PDGF receptor kinase family-oncogenic activation of v-kit involves deletion of extracellular domain and C terminus. EMBO (Eur. Mol. Biol. Organ.) J. 7:1003-1011.

4. Reith, A. D., and A. Bernstein. 1991. Molecular biology of the $W$ and Steel loci. In Genome Analysis. Volume 3. Genes and Phenotypes. Cold Spring Harbor Laboratory Press, Cold Spring Harbor, NY. 105-133.

5. Zsebo, K. M., J. Wypych, I. K. McNiece, H. S. Lu, K. A. Smith, S. B. Karkare, R. K. Sachdev, V. N. Yuschenkoff, N. C. Birkett, L. R. Williams, et al. 1990. Identification, purification, and biological characterization of hematopoietic stem cell factor from buffalo rat liver-conditioned medium. Cell. 63:195201.

6. Martin, F. H., S. V. Suggs, K. E. Langley, H. S. Lu, J. Ting, K. H. Okino, C. F. Morris, I. K. McNiece, F. W. Jacobson, E. A. Mendiaz, et al. 1990. Primary structure and functional expression of rat and human stem cell factor DNAs. Cell. 63:203-211.

7. Zsebo, K. M., D. A. Williams, E. N. Geissler, V. C. Broudy, F. H. Martin, H. L. Atkins, R. Y. Hsu, N. C. Birkett, K. H. Okino, D. C. Murdock, et al. 1990. Stem cell factor is encoded at the $S 1$ locus of the mouse and is the ligand for the c-kit tyrosine kinase receptor. Cell. 63:213-224.

8. Williams, D. E., J. Eisenman, A. Baird, C. Rauch, V. K. Ness, C. J. March, L. S. Park, U. Martin, D. Y. Mochizuki, H. S. Boswell, et al. 1990. Identification of a ligand for the c-kit proto-oncogene. Cell. 63:167-174.
9. Copeland, N. G., D. J. Gilbert, B. C. Cho, P. J. Donovan, N. A. Jenkins, D. Cosman, D. Anderson, S. D. Lyman, and D. E. Williams. 1990. Mast cell growth factor maps near the steel locus on mouse chromosome 10 and is deleted in a number of steel alleles. Cell. 63:175-183.

10. Anderson, D. M., S. D. Lyman, A. Baird, J. M. Wignall, J. Eisenman, C. Rauch, C. J. March, H. S. Boswell, S. D. Gimpel, D. Cosman, and D. E. Williams. 1990. Molecular cloning of mast cell growth factor, a hematopoietin that is active in both membrane bound and soluble forms. Cell. 63:235-243.

11. Nocka, K., J. Buck, E. Levi, and P. Besmer. 1990. Candidate ligand for the c-kit transmembrane kinase receptor. $\mathrm{KL}$, a fibroblast derived growth factor stimulates mast cells and erythroid progenitors. EMBO (Eur. Mol. Biol. Organ.) J. 9:3287-3294.

12. Huang, E., K. Nocka, D. R. Beier, T. Y. Chu, J. Buck, H. W. Lahm, D. Wellner, P. Leder, and P. Besmer. 1990. The hematopoietic growth factor KL is encoded by the $\mathrm{S} 1$ locus and is the ligand of the c-kit receptor, the gene product of the $W$ locus. Cell. 63:225-233.

13. Chabot, B., D. A. Stephenson, V. M. Chapman, P. Besmer, and A. Bernstein. 1988. The proto-oncogene c-kit encoding a transmembrane tyrosine kinase receptor maps to the mouse $W$ locus. Nature (Lond.). 335:88-89.

14. Geissler, E. N., M. A. Ryan, and D. E. Housman. 1988. The dominantwhite spotting $(W)$ locus of the mouse encodes the c-kit proto-oncogene. Cell. 55:185-192.

15. Russell, E. S. 1979. Hereditary anemia of the mouse: a review for geneticists. Adv. Gen. 20:357-459.

16. Kitamura, Y. 1989. Heterogeneity of mast cells and phenotypic change between subpopulations. Annu. Rev. Immunol. 7:59-76.

17. Galli, S. J., and Y. Kitamura. 1987. Genetically mast-cell-deficient $W / W^{v}$ and $S 1 / S 1^{d}$ mice. Am. J. Pathol. 127:191-198.

18. Denberg, J. A. 1992. Basophil and mast cell lineages in vitro and in vivo. Blood. 79:846-860.

19. Ogawa, M., Y. Matsuzaki, S. Nishikawa, S-I. Hayashi, T. Kunisada, T. Sudo, T. Kina, H. Nakauchi, and S-I. Nishikawa. 1991. Expression and function of c-kit in hematopoietic progenitor cells. J. Exp. Med. 174:63-71.

20. Papayannopoulou, T., M. Brice, V. C. Broudy, and K. M. Zsebo. 1991 Isolation of c-kit receptor-expressing cells from bone marrow, peripheral blood, and fetal liver: functional and composite antigenic profile. Blood. 78:1403-1412.

21. Ashman, L. K., A. C. Cambareri, L. B. To, R. J. Levinsky, and C. A. Juttner. 1991. Expression of the YB5.B8 antigen (c-kit proto-oncogene product) in normal human bone marrow. Blood. 78:30-37.

22. McNiece, I. K., K. E. Langley, and K. M. Zsebo. 1991. Recombinant human stem cell factor synergizes with GM-CSF, G-CSF, IL-3 and EPO to stimulate human progenitor cells of the myeloid erythroid lineages. Exp. Hematol. (NY). 19:226-231.

23. Migliaccio, G., A. R. Migliaccio, M. L. Druzin, P. J. V. Giardina, K. M. Zsebo, and J. W. Adamson. 1991. Effects of recombinant human stem cell factor (SCF) on the growth of human progenitor cells in vitro. J. Cell. Physiol. 148:503509.

24. Broxmeyer, H. E., S. Cooper, L. Lu, G. Hangoc, D. Anderson, D. Cosman, S. D. Lyman, and D. E. Williams. 1991. Effect of murine mast cell growth factor (c-kit proto-oncogene ligand) on colony formation by human marrow hematopoietic progenitor cells. Blood. 77:2142-2149.

25. Andre, C., L. d'Auriol, C. Lacombe, S. Gisselbrecht, and F. Galibert. 1989. c-kit mRNA expression in human and murine hematopoietic cell lines. Oncogene. 4:1047-1049.

26. Wang, C., J. E. Curtis, E. N. Geissler, E. A. McCulloch, and M. D. Minden. 1989. The expression of the proto-oncogene c-kit in the blast cells of acute myeloblastic leukemia. Leukemia (Baltimore). 3:699-702.

27. Kuriu, A., H. Ikeda, Y. Kanakura, J. D. Griffin, B. Druker, H. Yagura, H. Kitayama, J. Ishikawa, T. Nishiura, Y. Kanayama, et al. 1991. Proliferation of human myeloid leukemia cell line associated with the tyrosine-phosphorylation and activation of the proto-oncogene c-kit product. Blood. 78:2834-2840.

28. Ikeda, H., Y. Kanakura, T. Tamaki, A. Kuriu, H. Kitayama, J. Ishikawa, Y. Kanayama, T. Yonezawa, S. Tarui, and J. D. Griffin. 1991. Expression and functional role of the proto-oncogene c-kit in acute myeloblastic leukemia cells. Blood. 78:2962-2968.

29. Buhring, H-J, A. Ullrich, K. Schaudt, C. A. Muller, and F. W. Busch. 1991. The product of the proto-oncogene c-kit ( $\mathrm{P} 145^{\text {c-kit }}$ ) is a human bone marrow surface antigen of hematopoietic precursor cell which is expressed on a subset of acute nonlymphoblastic leukemia cells. Leukemia (Baltimore). 5:854860 .

30. Broudy, V. C., F. O. Smith, N. Lin, K. M. Zsebo, J. Egrie, and I. D. Bernstein. 1992. Blasts from patients with acute myelogenous leukemia express functional receptors for stem cell factor. Blood. 80:60-67.

31. Pietsh, Y., U. Kyas, U. Steffens, E. Yakisan, M. R. Hadam, Ludwig, K. M Zsebo, and K. Welte. 1992. Effect of human stem cell factor (c-kit ligand) on proliferation of myeloid leukemia cells: heterogeneity in response and synergy with other hematopoietic growth factors. Blood. 80:1199-1206.

32. Sillaber, C., D. Bevec, L. K. Ashman, J. H. Butterfield, K. Lechner, D. Maurer, P. Buttelheim, and P. Valent. 1992. IL-4 regulates c-kit gene product 
expression in human myeloid- and mast cell progenitors. J. Immunol. 147:42244228.

33. Kanakura, Y., B. Druker, S. A. Cannistra, Y. Furukawa, Y. Torimoto, and J. D. Griffin. 1990. Signal transduction of the human granulocyte-macrophage colony-stimulating factor and interleukin-3 receptors involves tyrosine phosphorylation of a common set of cytoplasmic proteins. Blood. 76:706-715.

34. Kanakura, Y., B. Drucker, J. Decarlo, S. A. Cannistra, and J. D. Griffin. 1991. Phorbol 12-myristate acetate 13-acetate inhibits granulocyte-macrophage colony-stimulating factor-dependent cell line. J. Biol. Chem. 266:490-496.

35. Lerner, N. B., K. H. Nocka, S. R. Cole, F. Qiu, A. Strife, L. K. Ashman, and P. Besmer. 1991. Monoclonal antibody YB5.B8 identifies the human c-kit protein product. Blood. 77:1876-1883.

36. Majumder, S., K. Brown, F-H. Qui, and P. Besmer. 1988. c-kit protein, a transmembrane kinase: identification in tissues and characterization. Mol. Cell. Biol. 8:4896-4903.

37. Butterfield, J. H., D. Weiler, G. Dewald, and G. J. Gleich. 1988. Establishment of an immature mast cell line from a patient with mast cell leukemia. Leuk. Res. 12:345-355.

38. Avanzi, G. C., P. Lista, B. Giovinazzo, R. Miniero, G. Saglio, G. Benetton, R. Coda, G. Cattoretti, and L. Pegoraro. 1988. Selective growth response to IL-3 of a human leukemic cell line with megakaryoblastic features. $\mathrm{Br}$. J. Haematol. 69:359-366.

39. Graham, F. L., J. Smith, W. C. Russell, and R. Nairn. 1977. Characteristics of a human cell line transformed by DNA from human adenovirus type $5 . J$. Gen. Virol. 36:59-72.

40. Tsujimura, T., S. Hirota, S. Nomura, Y. Niwa, M. Yamazaki, T. Tono, E. Morii, H. M. Kim, K. Kondo, Y. Nishimura, and Y. Kitamura. 1991. Characterization of Ws mutant allele of rats: a 12-base deletion in tyrosine kinase domain of c-kit gene. Blood. 78:1942-1946.

41. Tsujimura, T., U. Koshimizu, H. Katoh, Y. Isozaki, Y. Kanakura, T. Tono, S. Adachi, T. Kasugai, H. Tei, Y. Nishimune, et al. 1993. Mast cell number in the skin of heterozygotes reflects the molecular nature of c-kit mutation. Blood. $81: 2530-2538$.

42. Sanger, F., S. Nicklen, and A. R. Coulson. 1977. DNA sequencing with chain terminating inhibitors. Proc. Natl. Acad. Sci. USA. 74:5463-5467.

43. Mitsui, H., T. Furitsu, A. M. Dvorak, A-M. A. Irani, L. B. Schwartz, N. Inagaki, M. Takei, K. Ishizaka, K. M. Zsebo, S. Gillis, and T. Ishizaka. 1993. Development of human mast cells from umbilical cord blood cells by recombinant human and murine c-kit ligand. Proc. Natl. Acad. Sci. USA. 90:735-739.

44. Kunkel, T. A. 1985. Rapid and efficient site-specific mutagenesis without phenotypic selection. Proc. Natl. Acad. Sci. USA. 82:488-492.

45. Graham, F. L., and A. J. van der Eb. 1973. A new technique for the assay of infectivity of human adenovirus 5 DNA. Virology. 52:456-467.

46. Ullrich, A., and J. Schlessinger. 1990. Signal transduction by receptors with tyrosine kinase activity. Cell. 61:203-212.
47. Koch, C. A., D. Anderson, M. F. Moran, C. Ellis, and T. Pawson. 1991. SH2 domains: elements that control interactions of cytoplasmic signaling proteins. Science (Wash. DC). 252:668-674.

48. Rottapel, R., M. Reedijk, D. E. Williams, S. D. Lyman, D. M. Anderson, T. Pawson, and A. Bernstein. 1991. The steel/ $W$ transduction pathway: kit autophosphorylation and its association with a unique subset of cytoplasmic signaling proteins is induced by the steel factor. Mol. Cell. Biol. 11:3043-3051.

49. Reith, A. D., C. Ellis, S. D. Lyman, D. M. Anderson, D. E. Williams, A. Bernstein, and T. Pawson. 1991. Signal transduction by normal isoforms and $W$ mutant variants of the Kit receptor tyrosine kinase. EMBO (Eur. Mol. Biol. Organ.) J. 9:2451-2459.

50. Andre, C., E. Martin, F. Cornu, W-X. Hu, X-P. Wang, and F. Galibert. 1992. Genomic organization of the human c-kit gene: evidence of the receptor tyrosine kinase subclass III. Oncogene. 7:685-691.

51. Nocka, K., S. Majumder, B. Chabot, P. Ray, M. Cervone, A. Bernstein, and $P$. Besmer. 1989. Expression of c-kit gene products in known cellular targets of $W$ mutations in normal and $W$ mutant mice-evidence for an impaired c-kit kinase in mutant mice. Genes \& Dev. 3:816-826.

52. Yarden, Y., and A. Ullrich. 1988. Growth factor receptor tyrosine kinase. Annu. Rev. Biochem. 57:443-478.

53. Woolford, J. W., A. McAuliffe, and L. R. Rohrschneider. 1988. Activation of the feline c- $f m s$ proto-oncogene: multiple alterations are required to generate a fully transformed phenotype. Cell. 55:965-977.

54. Roussel, M., J. Downing, C. W. Rettenmier, and C. J. Sherr. 1988. A point mutation in the extracellular domain of the human CSF-1 receptor (c- $f m s$ protooncogene product) activates its transforming potential. Cell. 55:978-988.

55. Furitsu, T., H. Saito, A. M. Dvorak, L. B. Schwartz, A-M. A. Irani, J. F. Burdick, K. Ishizaka, and T. Ishizaka. 1989. Development of human mast cells in vitro. Proc. Natl. Acad. Sci. USA. 86:10039-10043.

56. Valent, P., E. Spanblochl, W. R. Sperr, C. Sillaber, K. M. Zsebo, H. Agis, H. Strobl, K. Geissler, P. Bettelheim, and K. Lechner. 1992. Induction of differentiation of human mast cells from bone marrow and peripheral blood mononuclear cells by recombinant human stem cell factor/kit-ligand in long-term culture. Blood. 80:2237-2245.

57. von Ruden, T., S. Kandels, T. Radaszkiewicz, A. Ullrich, and E. F. Wagner. 1992. Development of a lethal mast cell disease in mice reconstituted with bone marrow cells expressing the v-erbB oncogene. Blood. 79:3145-3158.

58. Sekido, Y., Y. Obata, R. Ueda, T. Hida, M. Suyama, K. Shimokata, Y. Ariyoshi, and T. Takahashi. 1991. Preferential expression of c-kit protooncogene transcripts in small cell lung cancer. Cancer Res. 51:2416-2419.

59. Strohmeyer, T., S. Peter, M. Hartmann, S. Munemitsu, R. Ackermann, A. Ullrich, and D. J. Slamon. 1991. Expression of the hst-1 and c-kit protooncogenes in human testicular germ cell tumors. Cancer Res. 51:1811-1816.

60. Hanks, S. K., A. M. Quinn, and T. Hunter. 1988. The protein kinase family: conserved features and deduced phylogeny of the catalytic domains. Science (Wash. DC). 241:42-52. 